Pacific Journal of Mathematic 


\title{
THE THEORY OF AD-ASSOCIATIVE LIE ALGEBRAS
}

\author{
Richard C. Penney
}

\begin{abstract}
A Lie algebra $\mathscr{L}$ is said to be ad-associative if the image of the adjoint representation of $\mathscr{L}$ on $\mathscr{L}$ is an associative algebra under composition. We show that every ad-associative Lie algebra is a quotient of a left commutative $(x y w=y x w)$ associative algebra by a Lie ideal. We conclude that every ad-associative Lie algebra is solvable and every irreducible representation of a nilpotent, ad-associative Lie group is square integrable modulo its kernel. We also characterize the $H A T$ algebras of Howe [2] in terms of associative algebra.
\end{abstract}

Let $\mathscr{L}$ be a finite dimensional Lie algebra over $\boldsymbol{R}$ and let ad denote the adjoint representation of $\mathscr{L}$ on $\mathscr{L}$. Let ad $\mathscr{L}$ be the image of ad. $\mathscr{L}$ is said to be ad-associative if ad $\mathscr{L}$ is closed under composition. In this case ad $\mathscr{L}$ is an associative algebra. Let us denote this algebra by $\mathscr{A}$. It is the purpose of this paper to give a structure theory for the ad-associative Lie algebras.

Our interest in the subject of ad-associative algebras stems from several different sources. The $H A T$ algebras introduced by Howe in [2] in connection with the study of ocillatory integrals can be shown to be ad-associative. In fact, we prove what we feel to be a very pretty characterization of the $H A T$ algebras. Ad-associative algebras also occur naturally in algebraic topology as a way of combining the information contained in the homology and co-homology groups of compact manifolds together. Here they give rise to some new topological invariants which are functions of the joint homology and cohomology groups (see Example II below). In another direction, there is a natural way of associating with any multi-linear form $\mathscr{B}$ on a vector space $\mathscr{V}$ an ad-associative Lie algebra $\mathscr{L}_{\mathscr{B}}$. Whether or not this association has any real significance remains to be seen. At the very least, the study of ad-associative algebras provides an interesting source of examples.

To begin our discussion, recall that any associative algebra $\mathscr{A}$ gives rise to a Lie algebra by setting $[x, y]=x y-y x$. If $\mathscr{L}$ is ad-associative, the Lie algebra corresponding to $\mathscr{A}=$ ad $\mathscr{L}$, is just $\mathscr{L} / \mathscr{Z}$ where $\mathscr{Z}$ is the center of $\mathscr{L}$. Hence $\mathscr{A}$ fits into the exact sequence of Lie algebras $0 \rightarrow \mathscr{Z} \rightarrow \mathscr{L} \rightarrow \mathscr{L} \rightarrow 0$. As a vector space $\mathscr{L}=\mathscr{A} \times \mathscr{z}$. In fact, there is an alternating, bi-linear mapping $\phi: \mathscr{A} \times \mathscr{A} \rightarrow \mathscr{Z}$ such that

$$
[(x, s),(y, t)]=([x, y], \dot{\phi}(x, y))
$$


for $x, y \in \mathscr{A}$ and $s, t \in \mathscr{z}$.

LEMMA. $\phi$ is nondegerate and satisfies $\phi([x, y], w)=-\phi(x, w y)$.

Proof. The nondegeneracy is obvious. The identity follows from the statement $[[z, y], w]=\operatorname{ad} w \operatorname{ad} y(x)$. Hence, if $x=\left(x_{1}, s\right), y=\left(y_{1}, t\right)$ and $w=\left(w_{1}, r\right)$ then ad $w$ ad $y(x)=\operatorname{ad}\left(w_{1} y_{1}, 0\right)(x)=\left(\left[w_{1} y_{1}, x_{1}\right], \phi\left(w_{1} y_{1}, x_{1}\right)\right)$. On the other hand $[[x, y], w]=\left(\left[\left[x_{1}, y_{1}\right], w_{1}\right], \phi\left(\left[x_{1}, y_{1}\right], w_{1}\right)\right.$.

DEFINITION. A twine algebra is a triple $(\mathscr{A}, \mathscr{L}, \phi)$ consisting of a finite dimensional associative algebra $\mathscr{A}$, a finite dimensional vector space $\mathscr{Z}$ and an alternating, nondegenerate, bi-linear mapping $\phi: \mathscr{A} \times \mathscr{A} \rightarrow \mathscr{Z}$ which satisfies

$$
\phi([x, y], w)=-\phi(x, w y)
$$

for all $x, y, w \in \mathscr{A}$. The above identity is called the twine identity.

In the above discussion, we defined a mapping of the category of ad-associative Lie algebras into the category of twine algebras. In the next lemma, we note that this mapping is surjective. If we wished to define morphisms of twine algebras, we could also prove functorality.

Proposition. Let $(\mathscr{A}, \mathscr{L}, \phi)$ be a twine algebra. Let $\mathscr{L}=$ $\mathscr{A} \times$ with the Lie algebra structure given by

$$
[(x, s),(y, t)]=([x, y], \phi(x, y)) .
$$

Then $\mathscr{L}$ is an ad-associative Lie algebra and ad $\mathscr{L} \approx \mathscr{A}$.

Proof. The ad-associativity is obvious once one knows that $\mathscr{L}$ is a Lie algebra. To show that $\mathscr{L}$ is a Lie algebra, one must show the Jacobi identity which amounts to showing that

$$
\phi(x,[y, w])=\phi([x, y], w)+\phi(y,[x, w]) .
$$

This follows trivially from the twine identity.

REMARK. Note that the above proof that $\mathscr{L}$ is a Lie algebra did not use the associativity of $\mathscr{C}$. Nonassociative twine algebras will be called quasi-twine algebras. The class of Lie algebras produced from quasi-twine algebras is interesting as in the nilpotent case it is precisely the class of Lie algebras with square integrable representations.

The following lemma is basic to the study of twine algebras. If $\mathscr{C}$ is a subset of a twine algebra $\mathscr{A}, \mathscr{C}^{\phi}$ will denote its 
orthogonal space relative to $\phi-$ i.e.,

$$
\mathscr{M}^{\phi}=\{x \in \mathscr{A} \mid \phi(\mathscr{L}, x)=\{0\}\} \text {. }
$$

Lemma A. Let $(\mathscr{A}, \mathscr{\varkappa}, \phi)$ be a twine algebra. Then

(a) $\phi(x y, w)=\phi(y, x w)$ for all $x, y, w \in \mathscr{A}$.

(b) $x y w=y x w$ for $x, y, w \in \mathscr{A}$.

(c) A subspace $\mathscr{I}$ of $\mathscr{A}$ is a left ideal of $\mathscr{A}$ iff $\mathscr{I}^{\phi}$ is a left ideal of $\mathscr{A}$. $\mathscr{I}$ is a right ideal iff $\mathscr{I}^{\phi}$ is a Lie ideal. $\mathscr{I}$ is a two sided ideal iff $\mathscr{J}^{\phi}$ is a two sided ideal.

(d) A mapping $T: \mathscr{A} \rightarrow \mathscr{A}$ which is a Lie algebra homomorphism and which preserves $\phi$ is an algebra isomorphism.

Proof. (a) follows by applying the twine identity to both sides of the equality

$$
\dot{\phi}(x,[y, w])=-\dot{\phi}(x,[w, y]) .
$$

(b) follows from (a) and associativity since

$$
\phi((x y) w, z)=\phi(w,(x y) z)=\phi(y(x w), z) .
$$

(c) is clear.

(d) is seen as follows. $T$ is invertible since it leaves $\phi$ invariant

$$
\begin{aligned}
\phi(T(x y), w) & =\phi\left(x y, T^{-1} w\right)=\phi\left(x,\left[T^{-1} w, y\right]\right) \\
& =\phi\left(x, T^{-1}[w, T y]\right)=\phi(T x T y, w)
\end{aligned}
$$

COROLlary. Let $\mathscr{L}$ be an ad-associative Lie algebra. Then $[\mathscr{L}, \mathscr{L}]$ is abelian, so $\mathscr{L}$ is solvable.

Proof. Let $\mathscr{A}=$ ad $\mathscr{L}$ and let $\mathscr{L}=\mathscr{A} \times \mathscr{Z}$ as above. [ $\mathscr{L}, \mathscr{L}]$ will be abelian if $[\mathscr{A}, \mathscr{A}]$ is abelian and $\phi$ is trivial on $[\mathscr{A}, \mathscr{A}] \times$ $[\mathscr{A}, \mathscr{A}]$. But from (b) above $[\mathscr{A}, \mathscr{A}]^{2}=0$ and from (b) and the twine identity

$$
\phi([x, y],[u, v])=\phi(y,[u, v] x)=0 .
$$

Property (b) above is referred to as left commutativity. Now, let $\mathscr{B}$ be any left commutative, associative algebra. It is easily seen that as a Lie algebra, $\mathscr{B}$ is ad-associative. In fact, for $x, y \in \mathscr{B},[x,[y, w]]=[x y, w]$. More generally, if $\mathscr{J}$ is any Lie ideal of $\mathscr{B}$, then the Lie algebra $\mathscr{L}=\mathscr{B} / \mathscr{J}$ is ad-associative. Our first main result is the following.

THeOREm I. Suppose $\mathscr{L}$ is an ad-associative Lie algebra. Then there is a left commutative algebra $\mathscr{B}$ and a Lie ideal $\mathscr{I}$ of $\mathscr{B}$ such that $\mathscr{L} \approx \mathscr{B} / \mathscr{I}$ as a Lie algebra. 
We prove this theorem by first constructing a class of "free" left commutative algebras for which the set of twine forms is explicitly describable. We then show that $\mathscr{A}=\mathscr{L} / \mathscr{Z}$ is the image of one of these free twine algebras.

Suppose we are given a family $\mathscr{N}_{1}, \cdots, \mathscr{N}_{n}$ of abelian associative algebras. Our first step is to construct a left commutative algebra $\mathscr{B}$ which contains the $\mathscr{N}_{i}$ as subalgebras and which is "freely" generated by the $\mathscr{N}_{i}$. The most natural construction of such an algebra would be to form all possible tensor products of all lengths of the $\mathscr{N}_{i}$, take a direct sum, define a multiplication and then quotient out by an ideal. Unfortunately this obvious approach is very complicated notationally. It turns out to be much simpler if one first adjoins units onto each of the spaces $\mathscr{N}_{i}$ for then we can work with only $n$-fold tensor products.

Specifically, let $\mathscr{A}_{i}$ be the algebras defined on $\boldsymbol{R} \times \mathscr{N}_{i}$ by

$$
(s, a)(t, b)=(s t, s b+t a) .
$$

Let $\sigma_{n}$ be the set of permutations of the set $\{1, \cdots, n\}$. For $\pi \in \sigma_{n}$, let $\mathscr{A}_{\pi}=\mathscr{A}_{n(1)} \otimes \cdots \otimes \mathscr{A}_{\pi(n)}$.

Let

$$
P=P\left(\mathscr{A}_{1}, \cdots, \mathscr{A}_{n}\right)=\sum \oplus \mathscr{A}_{\pi} \quad\left(\pi \in \sigma_{n}\right) .
$$

If $a$ is a sequence indexed by $\{1, \cdots, n\}$ such that $a(i) \in \mathscr{A}_{i}$ for all $i$ we set $a \pi=a(\pi(1)) \otimes \cdots \otimes a(\pi(n)) \in \mathscr{A}_{\pi}$ for all $\pi \in \sigma_{n}$. We define an algebra structure on $P$ by setting $a_{\pi} b_{\tau}=a(\tau(1)) b(\tau(1)) \otimes \cdots \otimes$ $a(\tau(n)) b(\tau(n))$ for all sequences $a$ and $b$ as above. We extend this multiplication by distributivity to all of $P$. It is easily seen that under this multiplication $P$ becomes an associative, noncommutative algebra (But note that $P$ is always left commutative). We shall refer to $P$ as the $n$-pole algebra of $\mathscr{A}_{1}, \cdots, \mathscr{A}_{n}$. The elements of set $\mathscr{A}_{\pi}$ are called $\pi$ oriented. If $a$ is a sequence such that $a(i) \in \mathscr{A}_{i}$ for all $i$, then $a_{\pi}$ is the $\pi$ orientation of $a$. The reason for this terminology is that the way one multiplies $a_{\pi}$ and $b_{\tau}$ is to change the orientation of $a_{\pi}$ so that it agrees with that of $b_{\tau}$ and then take a standard product. This seems analogous to magnetic dipoles lining up to form a new dipole.

We may consider $\mathscr{N}_{i}$ as the subalgebra $0 \times \mathscr{N}_{i}$ of $\mathscr{A}_{i}$. For each $i$ let $1_{i}=(1,0)$ considered as an element of $\mathscr{A}_{i}\left(0\right.$ is the zero of $\left.\mathscr{N}_{i}\right)$. We embed $\mathscr{N}_{i}$ into $P\left(\mathscr{A}_{1}, \cdots, \mathscr{A}_{n}\right)$ as the subalgebra $1_{1} \otimes 1_{2} \otimes \cdots \otimes$ $1_{\hat{i}} \otimes \cdots \otimes 1_{n} \otimes \mathscr{N}_{i} . \quad$ Let $L C\left(\mathscr{N}_{1}, \cdots, \mathscr{N}_{n}\right)$ be the subalgebra of $P\left(\mathscr{A}_{1}\right.$, $\left.\cdots, \mathscr{A}_{n}\right)$ generated by $\left\{\mathscr{N}_{i}\right\}$ embedded as above. $L C$ is by definition the left commutative algebra freely generated by $\mathscr{N}_{1}, \cdots, \mathscr{N}_{n}$.

Our main theorem concerning freely generated left commutative algebras is the following. 
Let $\mathscr{\varkappa}$ be a finite dimensional vector space.

Proposition. Let $\left\{\mathscr{N}_{i}\right\}$ be a finite family of abelian associative algebras and let $\phi$ be a $\mathscr{Z}$ valued twine form on $L C\left(\mathscr{N}_{1}, \cdots, \mathscr{N}_{n}\right)$ which is zero on each $\mathscr{N}_{i} \times \mathscr{N}_{i}$. Then there is a linear mapping $\alpha$ of $L C$ into $\mathscr{z}$ such that $\phi(x, y)=\alpha([x, y])$ for all $x, y \in L C$.

Proof. We begin with notation. Let $\tau_{i}$ denote the embedding of $\mathscr{N}_{i}$ in $L C\left(\mathscr{N}_{1}, \cdots, \mathscr{N}_{n}\right)$ used in defining $L C$-i.e., $\tau_{i}(a)=1_{1} \otimes$ $\cdots 1_{\hat{1}} \otimes \cdots \otimes 1_{n} \otimes a$.

Let $S$ be an arbitrary nonempty subset of $\{1, \cdots, n\}$ and let $a \in \Pi_{s} \mathscr{N}_{i}$ (the product set). Let $n=\left(i_{1}, \cdots, i_{k}\right)$ be an enumeration of the elements of $S$. We shall define $a(S, n)=\tau_{i_{1}}\left(a\left(i_{1}\right)\right) \cdots \tau_{i}(a(i)) \in$ $L C\left(\mathscr{N}_{1}, \cdots, \mathscr{N}_{n}\right)$. By left commutativity $a(S, n)$ depends only on $i_{k}$ and not on the specific enumeration $n$. Hence we may write $a(S, n)=$ $a\left(S, i_{k}\right)$. By definition, $L C$ is the span of the $a(S, j)$ with $S, j$ and $a$ varying. Note that for $a \in \Pi_{S} \mathscr{N}_{i}$ and $b \in \Pi_{T} \mathscr{N}_{i}$ where $S, T \subset$ $\{1, \cdots, n\}$, we have the identity $a(S, i) b(T, j)=c(S \cup T, j)$ where $c$ is the sequence on $S \cup T$ given by $c(k)=a(k)$ for $k \in S \sim T, c(k)=$ $b(k)$ for $k \in T \sim S$ and $c(k)=a(k) b(k)$ for $k \in S \cap T$. For each $S \subset$ $\{1, \cdots, n\}$ and each $j \in S$, let $\mathscr{N}(S, j)$ be the span of the elements $a(S, i)$ as above. Then

$$
L C\left(\mathscr{N}_{1}, \cdots, \mathscr{N}_{n}\right)=\sum \oplus \mathscr{N}(S, j) \quad(S \subset\{1, \cdots, n\}, j \in S) .
$$

Now let $\phi$ be a twine form on $L C\left(\mathscr{N}_{1}, \cdots, \mathscr{N}_{n}\right)$. We shall define mappings $\alpha(S, i)$ on $\mathscr{N}(S, i)$ such that $\phi(x, y)=\alpha([x, y])$ where $\alpha=$ $\sum_{S, i} \alpha(S, i)$. First, however let $S \subset\{1, \cdots, n\}$ and let $a \in \Pi_{s} \mathscr{N}_{i}$. Suppose that there are two elements $i$ and $j$ in $S$ with $i \neq j$. Let $S=S_{1} \cup S_{2}$ be a partitioning of $S$ with $i \in S_{1}$ and $j \in S_{2}$. Let

$$
\beta(i, j, S, a)=\phi\left(a\left(S_{1}, i\right), \quad a\left(S_{2}, j\right)\right) .
$$

In principle $\beta$ depends also on the partitioning $S_{1}, S_{2}$ of $S$. However, the left commutativity of $L C$ and the left self-adjointness of twine forms imply that $\beta$ is in fact independent of the partitioning of $S$. We extend the definition of $\beta$ to the cases where $S$ is a singleton set or $i=j$ by setting $\beta(j, j, S, a)=0$.

LEMMA. $\beta$ is skew-symmetric in $i$ and $j$ and satisfies the cocycle identity:

$$
\begin{array}{r}
\beta(i, j, S, a)+\beta(j, k, S, a)=\beta(i, k, S, a) \\
\text { for all } i, j, k \in S .
\end{array}
$$

Proof. The skew symmetry is obvious. 
The co-cycle identity is trivial if any of the elements $\{i, j, k\}$ are equal so we will assume that $i, j$ and $k$ are distinct. In this case there is a partitioning $S=S_{1} \cup S_{2} \cup S_{3}$ with $i \in S_{1}, j \in S_{2}$ and $k \in S_{3}$. From the identity

$$
\left[a\left(S_{2}, j\right), a\left(S_{1}, i\right)\right]=a\left(S_{1} \cup S_{2}, i\right)-a\left(S_{1} \cup S_{2}, j\right)
$$

we conclude

$$
\begin{aligned}
\beta(i, k, S, a)-\beta(j, k, S, a) & =\phi\left(\left[a\left(S_{2}, j\right), a\left(S_{1}, i\right)\right], a\left(S_{3}, k\right)\right) \\
& =\phi\left(a\left(S_{1}, i\right), a\left(S_{3}, k\right) a\left(S_{2}, j\right)\right) \\
& =\phi\left(a\left(S_{1}, i\right), a\left(S_{2} \cup S_{3}, j\right)\right) \\
& =\beta(i, j, S, a) .
\end{aligned}
$$

Now the lemma implies that there are multi-linear mappings $\tilde{\alpha}(S, k): \prod_{S} \mathscr{N}_{i} \rightarrow \mathscr{Z}$ such that $\beta(i, j, S, a)=\tilde{\alpha}(S, i)(a)-\tilde{\alpha}(S, j)(a)$. To see this let $k_{0} \in S$ be fixed and let $\tilde{\alpha}(S, i)(a)=\beta\left(i, k_{0}, S, a\right)$. Since $\mathscr{N}(S, i)$ is isomorphic with $\otimes \mathscr{N}_{i}$, we may define linear mappings $\alpha(S, i)$ on $\mathscr{N}(S, i)$ by setting

$$
\alpha(S, i)(a(S, i))=\tilde{\alpha}(S, i)(a)
$$

for all $a \in \Pi_{s} \mathscr{N}_{i}$. We then extend $\alpha(S, i)$ linearly to all of $\mathscr{N}(S, i)$. Let $\alpha: L C\left(\mathscr{N}_{1}, \cdots, \mathscr{N}_{n}\right) \rightarrow \mathscr{Z}$ be defined by $\alpha \mid \mathscr{N}(S, i)=\alpha(S, i)$.

To finish our theorem we need to show that $\phi(x, y)=\alpha([x, y])$. To prove this it suffices to consider $x=a(S, i)$ and $y=b(T, j)$ for some $S, T \subset\{1, \cdots, n\}$ and $i \in S, j \in T$. Let $c$ be the element of $\Pi_{S \cup T} \mathscr{N}_{k}$ by $c=a$ on $S \sim T, c=b$ on $T \sim S$ and $c(j)=a(j) b(j)=$ $b(j) a(j)$ on $S \cap T$. From the left self-adjointness of $\phi$ and let commutativity we see easily, for $i \neq j$,

$$
\begin{aligned}
\phi(a(S, i), b(T, j)) & =\beta(i, j, S \cup T, c) \\
& =\tilde{\alpha}(i, S \cup T, c)-\tilde{\alpha}(j, S \cup T, c) \\
& =\alpha(c(S \cup T, i))-\alpha(c(S \cup T, j)) \\
& =\alpha([a(S, i), b(T, j)]) .
\end{aligned}
$$

If $i=j, \phi(a(S, i), b(T, i))=0$ since in this case $a(S, i)$ and $b(T, i)$ both belong either to $(L C) \mathscr{N}_{i}$ or to $\mathscr{N}_{i}$, and, for example $\phi(x a, y b)=$ $\phi(y x,[a, b])=0$ for all $a$ and $b$ in $\mathscr{N}_{i} \cdot \mathscr{N}_{i} \cdot \mathscr{N}_{i}+(L C) \mathscr{N}_{i}$ is abelian so $\phi_{\alpha}$ is zero on $\mathscr{N}_{i}+(L C) \mathscr{N}_{i}$ also.

Our theorem follows from the above proposition as follows. Let $\mathscr{A}=\mathscr{L} / \mathscr{Z}$ and let $\phi: \mathscr{A} \times \mathscr{A} \rightarrow \mathscr{Z}$ be the twine form. Let $\mathscr{N}_{1}, \cdots, \mathscr{N}_{i}$ be a family of abelian subalgebras of $\mathscr{A}$ such that $\phi$ is trivial on $\mathscr{N}_{i} \times \mathscr{N}_{i}$ and $\mathscr{N}_{i}$ generate $\mathscr{A}$. Such $\mathscr{N}_{i}$ exist. For 
example, if $e_{1}, \cdots, e_{n}$ generate $\mathscr{A}$ as a left $\mathscr{A}$ module, let $\mathscr{N}_{i}=$ $\mathscr{A} e_{i}$.

It is easily verified that there is an algebra homomorphism $T$ of $L C\left(\mathscr{N}_{1}, \cdots, \mathscr{N}_{k}\right)$ onto $\mathscr{A}$. Let $\tilde{\phi}$ be the pull back of $\phi$ by $T$ to $L C \times L C$. From the proposition there is a mapping $\alpha: L C \rightarrow \mathscr{\&}$ such that $\alpha([x, y])=\tilde{\phi}(x, y)$.

We define an algebra structure on $\mathscr{B}=L C \times \mathscr{Z}$ by setting

$$
(x, v) \cdot(y, w)=(x y, \alpha(x y)) \text {. }
$$

Under this structure, $\mathscr{B}$ becomes a left commutative algebra. We extend the mapping $T: L C \rightarrow \mathscr{A}$ to a mapping $\widetilde{T}: \mathscr{B} \rightarrow \mathscr{L}=\mathscr{A} \times$ $\mathscr{Z}$ by setting $\widetilde{T}(x, v)=(T x, v) . \quad \widetilde{T}$ is easily seen to be a surjective Lie algebra homomorphism.

The proof of the above theorem also provides us with a general construction capable of producing all twine algebras. To see this, let $\mathscr{N}_{1}, \cdots, \mathscr{N}_{k}$ be any family of abelian algebras. Let $\mathscr{Z}$ be a vector space and let $\alpha: L C\left(\mathscr{N}_{1}, \cdots, \mathscr{N}_{k}\right) \rightarrow \mathscr{Z}$ be any linear mapping. Then the form $\phi_{\alpha}(x, y)=\alpha([x, y])$ is a twine form on $L C$. It follows from Lemma A that the radical $\mathscr{R}_{\alpha}$ of $\phi_{\alpha}$ is an algebra ideal in $L C$.

Let

$$
T W\left(\mathscr{N}_{1}, \cdots, \mathscr{N}_{k}, \alpha\right)=L C\left(\mathscr{N}_{1}, \cdots, \mathscr{N}_{k}\right) / \mathscr{R}_{\alpha},
$$

and let $\phi$ be the projection of $\phi_{\alpha}$ to $T W$. Then the pair $(T W, \phi)$ is a twine algebra and every twine algebra is isomorphic with such a pair. Note that $\alpha$ is uniquely determined by its restriction to $\mathscr{N}(S, i)$ (see the above proof for notation) for $S \subset\{1, \cdots, n\}$ and $i \in S$. Also $\mathscr{N}(S, i) \approx \otimes_{i \in S} \mathscr{N}_{i}$ so $\alpha \mid \mathscr{N}(S, i)=\alpha(S, i)$ may be thought of as a multi-linear functional on $\prod_{i \in S} \mathscr{N}_{j}$. Hence a twine algebra is determined by giving for each subset $S$ of $\{1, \cdots, n\}$, a family $\left\{\alpha_{i}\right\}$ of multi-linear functionals on $\Pi_{j \in S} \mathscr{N}_{j}$ indexed by $S$. A particularly interesting case arises when $\alpha(S, i)=0$ if $S \neq\{1, \cdots, n\}$. In this case the necessary data is simply a family $\alpha_{1}, \cdots, \alpha_{n}$ of $n$, multilinear functionals on $\prod_{i=1}^{n} \mathscr{N}_{i}$. We consider several examples of this construction below.

ExAMPLE I. Let $\mathscr{S}$ be an abelian, nilpotent associative algebra and let $\mathscr{S}^{*}$ be its linear dual. Let $\alpha_{1}: \mathscr{S} \times \mathscr{S}^{*}$ be the functional.

$$
\alpha_{1}(x, y)=\langle x, y\rangle
$$

and let $\alpha_{2}=0$. Let $\mathscr{S}^{*}$ be considered as an algebra with trivial multiplication. Then 
$L C\left(\mathscr{S}, \mathscr{S}^{*}\right)=\left(1_{1} \otimes \mathscr{S}^{*}\right) \oplus\left(1_{2} \otimes \mathscr{S}\right) \oplus \mathscr{S} \otimes \mathscr{S}^{*} \oplus \mathscr{S}^{*} \otimes \mathscr{S}$.

The space $\mathscr{S}^{*} \otimes \mathscr{S}$ is central in $L C\left(\mathscr{S}_{,} \mathscr{S}^{*}\right)$ so it belongs to the radical $R_{\alpha}$ of $\phi_{\alpha}$.

Let $y \in \mathscr{S}^{*}$ and $x \in \mathscr{S}_{\text {. Define }} x \cdot y \in \mathscr{S}^{*}$ to be $\langle w, x \cdot y\rangle=\langle x w, y\rangle$. It is easily seen that $x \otimes y-1_{1} \otimes x y \in R_{\alpha}$ for all $x \in \mathscr{S}, y \in \mathscr{S}^{*}$.

It follows that

$$
T W\left(\mathscr{S}, \mathscr{S}^{*}\right) \approx \mathscr{S} \oplus \mathscr{S}^{*}
$$

with the product

$$
(x, y) \cdot\left(x^{\prime}, y^{\prime}\right)=\left(x x^{\prime}, x y^{\prime}\right) .
$$

In this case $\phi_{\alpha}$ projects to the form

$$
\phi\left((x, y),\left(x^{\prime}, y^{\prime}\right)\right)=\left\langle x, y^{\prime}\right\rangle-\left\langle x^{\prime}, y\right\rangle .
$$

Let $\mathscr{L}$ denote the ad-associative Lie algebra corresponding to the pair $\left(T W\left(\mathscr{S} \mathscr{S}^{*}\right), \phi\right)$. Such Lie algebras are known in the literature. They have been extensively studied by Howe in [2]. They are precisely the split $H A T$ algebras. Let us observe that in this case, the Lie algebra $\mathscr{L}$ itself comes from a left commutative algebra. In fact, we define a product structure on $\left(\mathscr{S} \otimes \mathscr{S}^{*}\right) \times \boldsymbol{R}$ by setting

$$
\left(\left(x_{1}, y_{1}\right), s\right)\left(\left(x_{2}, y_{2}\right), t\right)=\left(\left(x_{1} x_{2}, x_{1} y_{2}\right),\left\langle x_{1}, y_{2}\right\rangle\right) \text {. }
$$

Then $\left(\mathscr{S} \oplus \mathscr{S}^{*}\right) \times \boldsymbol{R}$ becomes a left commutative algebra which defines $\mathscr{L}$.

EXAMPLE II. This example is essentially an example of Example I. Let $M$ be a compact, orientable, $n$ dimensional manifold. Let $H_{i}(M, \boldsymbol{R})$ and $H^{i}(M, \boldsymbol{R})$ denote respectively the $i$ th homology and co-homology groups of $M$ over $\boldsymbol{R}$. Let

$$
\begin{aligned}
& H^{*}(M, \boldsymbol{R})=\sum_{i} \oplus H^{i}(M, \boldsymbol{R}) \\
& H_{*}(M, \boldsymbol{R})=\sum_{i} \oplus H_{i}(M, \boldsymbol{R}) .
\end{aligned}
$$

$H^{*}(M, \boldsymbol{R})$ is an algebra under the cup product. There is a pairing $\langle$,$\rangle between H^{i}(M, \boldsymbol{R})$ and $H_{i}(M, \boldsymbol{R})$ which makes $H_{i}(M, \boldsymbol{R})$ isomorphic with the dual space of $H^{i}(M, \boldsymbol{R})$. Hence $H_{*}(M, \boldsymbol{R})$ is cannonically isomorphic with the dual of $H^{*}(M, R)$. Let $\langle$,$\rangle denote the pairing.$ There is also a cup product operation

$$
\cap: H^{*}(M, \boldsymbol{R}) \times H_{*}(M, \boldsymbol{R}) \longrightarrow H_{*}(M, \boldsymbol{R})
$$

characterized by 


$$
\langle a, b \cap \xi\rangle=\langle a \cup b, \xi\rangle
$$

for $a, b \in H^{*}(M, \boldsymbol{R})$ and $\xi \in H_{*}(M, \boldsymbol{R})$. Let $\mathscr{B}=H^{*}(M, \boldsymbol{R}) \times H_{*}(M, \boldsymbol{R})$. We make $\mathscr{B}$ into an algebra by setting

$$
(a, m)(b, n)=(a \cup b, a \cap n) \text {. }
$$

$\mathscr{B}$ is referred to as the full co-homology algebra of $M$. It is obviously similar to the algebra considered in Example $I$, the main dissimularity being that $H^{*}(M, \boldsymbol{R})$ is nonabelian. This dissimularity can be eliminated by using

$$
H^{e}(M, \boldsymbol{R})=\sum \oplus H^{2 P}(M, \boldsymbol{R})
$$

and

$$
H_{e}(M, \boldsymbol{R})=\sum \oplus H_{2 P}(M, \boldsymbol{R})
$$

in place of $H^{*}(M, \boldsymbol{R})$ and $H_{*}(M, \boldsymbol{R})$ in the definition of $\mathscr{B}$. This is called the half full co-homology algebra. This replacement is especially pertinent if $n=\operatorname{dim} M$ is odd for in this case each $H^{P}(X, \boldsymbol{R})$ and each $H_{P}(X, \boldsymbol{R})$ appears once in $\mathscr{B}$ as $H^{P}(X, \boldsymbol{R}) \approx H_{n-P}(X, \boldsymbol{R})$ by Pontriagin duality. One can also use the integral homology to define discrete, co-compact subgroups of $(\mathscr{B}, *)$. One can then associate with $M$ a compact nilmanifold. Hence we have a functorial mapping of the category of compact orientable manifolds to the class of compact nilmanifolds. It seems that it might be quite interesting to study this functor.

EXAMPLE III. The algebra associated with a family of multilinear forms.

Let $V$ be a finite dimensional vector space over $\boldsymbol{R}$ and let $\left\{\alpha_{1}, \cdots, \alpha_{n}\right\}$ be a set of $n, n$-linear forms mapping $V^{n} \rightarrow \boldsymbol{R}$. We consider $V$ as an algebra with trivial multiplication. As discussed above Example I, we may use the forms $\alpha_{i}$ to define a functional $\alpha$ on $L C(V, \cdots, V)$ and hence construct $T W(V, \cdots, V, \alpha)$. If we are given fewer than $n$ forms (say one) we may form an algebra by declaring the rest of the $\alpha_{i}$ to be zero. It is clear that re-indexing the forms $\alpha_{i}$ does not change the isomorphism class of the algebra. To get specific, let $\alpha_{3}$ be the determinant function on $\boldsymbol{R}^{3} \times \boldsymbol{R}^{3} \times \boldsymbol{R}^{3}$ and let $\alpha_{1}$ and $\alpha_{2}$ be zero. We shall compute the corresponding twine algebra.

Let $V_{1}=V_{2}=V_{3}=\boldsymbol{R}^{3}$. The order-3 tensor products in $L C\left(V_{1}, V_{2}, V_{3}\right)$ are just the sets 
with $i<j$ and $k \notin\{i, j\}$. Each such set is central in $L C$ so they go to zero. The order two tensor products are spanned by the $1_{1} \otimes$ $V_{3} \otimes V_{2}, 1_{2} \otimes V_{3} \otimes V_{1}, 1_{1} \otimes V_{2} \otimes V_{3}, V_{1} \otimes 1_{2} \otimes V_{3}, V_{1} \otimes 1_{3} \otimes V_{2}, V_{2} \otimes$ $1_{3} \otimes V_{1}$.

The first of these sets goes to zero in $T W$ as the only tensor products which it doesn't centralize in $L C$ are those in $1_{2} \otimes 1_{3} \otimes V_{1}$ and $\left[1_{2} \otimes 1_{3} \otimes V_{1}, 1_{1} \otimes V_{3} \otimes V_{2}\right] \subset V_{1} \otimes V_{3} \otimes V_{2}+V_{2} \otimes V_{3} \otimes V_{1} \subset \operatorname{ker} \alpha$. Similarly for the second set. To study the third set, we note the identity. that

$$
\operatorname{Det}(u, v, w)=u \cdot(v \times w)=(u \times v) \cdot w \text { on } \boldsymbol{R}^{3} \times \boldsymbol{R}^{3} \times \boldsymbol{R}^{3} . \quad \text { It follow }
$$

$$
\alpha\left(\left[1_{1} \otimes v_{2} \otimes v_{3}, 1_{2} \otimes 1_{3} \otimes v_{1}\right]\right)=-v_{1} \cdot\left(v_{2} \times v_{3}\right) .
$$

Hence, the image of $1_{1} \otimes V_{2} \otimes V_{3}$ in $T W$ is isomorphic with $\boldsymbol{R}^{3}$ under the mapping $1_{1} \otimes v_{2} \otimes v_{3} \rightarrow v_{2} \times v_{3}$. Similar comments hold for the sets $V_{1} \otimes 1_{2} \otimes V_{3}, V_{1} \otimes 1_{3} \otimes V_{2}$ and $V_{2} \otimes 1_{3} \otimes V_{1}$. However, the latter two spaces are also identified with each other under the mapping

$$
v_{2} \otimes 1_{3} \otimes v_{1} \longrightarrow v_{1} \otimes 1_{3} \otimes v_{2} \text { in } T W .
$$

The order one tensor products are all independent.

Hence

$$
T W\left(\boldsymbol{R}^{3}, \boldsymbol{R}^{3}, \boldsymbol{R}^{3}, \text { det }\right)=\left(\boldsymbol{R}^{3}\right)^{3} \times\left(\boldsymbol{R}^{3}\right)^{3} .
$$

The product structure is:

$$
\begin{aligned}
& \left(v_{1}, v_{2}, v_{3}, w_{1}, w_{2}, w_{3}\right) \cdot\left(v_{1}^{\prime}, v_{2}^{\prime}, v_{3}^{\prime}, w_{1}^{\prime}, w_{2}^{\prime}, w_{3}^{\prime}\right) \\
& \quad=\left(0,0,0, v_{2} \times v_{3}^{\prime}, v_{1} \times v_{3}^{\prime}, v_{1} \times v_{2}^{\prime}-v_{2} \times v_{1}^{\prime}\right) \text { for } v_{i}, v_{i}^{\prime}, w_{i}, w_{i}^{\prime} \in \boldsymbol{R}^{3} .
\end{aligned}
$$

In [2], Howe introduced a concept of split $H A T$ lie algebra. Howe's definition is:

Definition. A nilpotent Lie algebra $\mathscr{V}$ is split $H A T$ if

(1) The dimension of the center $\mathscr{R}(\mathscr{N})$ is one.

(2) There are abelian subalgebras $\mathscr{S}$ and $\mathscr{C l}$ of $\mathscr{N}$ such that:

(a) $\mathscr{S} \cap \mathscr{H}=\mathscr{L}(\mathscr{N})$

(b) $\mathscr{N}=\mathscr{S}+\mathscr{L}$

(c) $\mathscr{L} \supset[\mathscr{N}, \mathscr{N}]$

(d) $\operatorname{dim} \mathscr{C l}=\operatorname{dim} \mathscr{S}$

(3) $\mathscr{N}$ has square integrable representations in the sense of Moore-Wolf.

Howe showed that $\mathscr{N}$ is split HAT iff $\mathscr{N}$ is Lie algebra isomorphic with an algebra of the form of Example I above. It follows from Example I that every split $H A T$ algebra is isomorphic with the Lie algebra defined by a left commutative, associative algebra with 
one dimensional center. In our next theorem, we prove a converse to this statement.

THeOREM II. A Lie algebra $\mathscr{N}$ is split, HAT iff $\mathscr{N}$ is Lie algebra isomorphic with the Lie algebra defined by a left commutative, associative, nilpotent algebra $\mathscr{B}$ with one dimensional center $\mathscr{Z}(\mathscr{B})$.

Proof. The existence of $\mathscr{B}$ was proven in Example I above, so we need only show that any such $\mathscr{B}$ is split $H A T$.

LEMMA. $\mathscr{Z}(\mathscr{B})$ annihilates $\mathscr{B}$ on both the left and the right.

Proof. Suppose not. Then there is an $n$ such that

$$
\mathscr{B}^{n} \mathscr{Z}(\mathscr{B}) \neq 0=\mathscr{B}^{n+1} \mathscr{2}(\mathscr{B}) \text {. }
$$

But then $\mathscr{B}^{n} \mathscr{Z}(\mathscr{B})$ is central so $\mathscr{B}^{n} \mathscr{Z}(\mathscr{B})=\mathscr{Z}(\mathscr{B})$. Hence $\mathscr{z}(\mathscr{B}) \mathscr{B}=\mathscr{B}^{n+1} \mathscr{Z}(\mathscr{B})=0$.

Let $\mathscr{A}=\mathscr{B} / \mathscr{Z}(\mathscr{B})$. As a vector space $\mathscr{B}=\mathscr{A} \times \mathscr{Z}(\mathscr{B})$. There is a form $\alpha: \mathscr{A} \times \mathscr{A} \rightarrow \mathscr{L}(\mathscr{B})$ such that

$$
(a, s)(b, t)=(a b, \alpha(a, b))
$$

for $a, b \in \mathscr{A}, s, t \in \mathscr{Z}(\mathscr{B})$. Let $\phi(a, b)=\alpha(a, b)-\alpha(b, a)$ for $a, b \in \mathscr{A}$. It is easily verified that $\phi$ is a twine for the algebra $\mathscr{A}$.

Lemma. $\phi$ is nondegenerate on $\mathscr{A} \times \mathscr{A}$.

Proof. Suppose $\phi(\alpha, x)=0$ for all $x \in \mathscr{A}$. Then

$$
[(x, t),(a, 0)]=([x, a], \phi(x, a))=([x, a], 0) .
$$

It follows that $[(x, t),(a, 0)] \in \mathscr{Z}(\mathscr{B})$ implies that $[(x, t),(a, 0)]=0$. But if the lemma is false, there is an $n$ such that $\left[\mathscr{P}^{n},(a, 0)\right]=0$ and $\left[\mathscr{B}^{n-1},(a, 0)\right] \neq 0$. This is impossible since, by left commutativity, $[x,[y,(a, 0)]]=[x y,(a, 0)]$. Hence $\left[\mathscr{B},\left[\mathscr{B}^{n-1},(a, 0)\right]\right]=\left[\mathscr{B}^{n},(a, 0)\right]=0$. Thus $\left[\mathscr{B}^{n-1},(a, 0)\right] \subset \mathscr{z}(\mathscr{B})$ and so is zero.

COROLLARY. The Lie group corresponding to $\mathscr{B}$ has square integrable representations in the sense of [4].

Proof. Let $Z_{0} \in \mathscr{Z}(\mathscr{B}), Z_{0} \neq 0$. Let $\lambda \in \mathscr{B}^{*}$ be the functional which maps $\left(a, t Z_{0}\right)$ into $t$. The radical of the form $\phi_{\lambda}$ on $\mathscr{B} \times \mathscr{B}$ defined by

$$
\phi_{\lambda}(x, y)=\lambda([x, y])
$$


is $\mathscr{Z}(\mathscr{B})$ as follows from the above lemma. From Moore-Wolf [4], this is sufficient to prove the existence of square integrable representations.

Now from the nondegeneracy of $\phi$ on $\mathscr{A} \times \mathscr{A}$, there is a unique linear mapping $\tau: \mathscr{A} \rightarrow \mathscr{A}$ such that $\alpha(x, y)=\phi(\tau x, y)$. The splitting of $\mathscr{B}$ will be defined from the generalized eigenspaces of $\tau$. First we need some information concerning $\tau$.

LEMma. $\tau$ is a left module homomorphism of $\mathscr{A}$ into $\mathscr{A}$ which is trivial on [\&, $\mathscr{A}]$. The adjoint of $\tau$ relative to $\phi$ is $\tau^{\phi}=I-\tau$ and $\tau^{\phi}$ maps $\mathscr{A}$ into the left annihilator $\mathscr{L}(\mathscr{A})$ of $\mathscr{A}$.

Proof. The equality

$$
\phi(x, y)=\alpha(x, y)-\alpha(y, x)=\phi(\tau x, y)-\dot{\phi}(\tau y, x)
$$

implies the adjoint statement. The triviality of $\tau$ on $[\mathscr{A}, \mathscr{A}]$ follows from the left commutativity of $\mathscr{B}$ for in $\mathscr{B},(0,0)=[(x, 0),(y, 0)]$ $(w, 0)=(0, \alpha([x, y], w))$. The left module homomorphism property follows similarly from

$$
x(y w)=(y x) w
$$

in $\mathscr{B}$. The fact that $\tau^{\phi}$ maps into $\mathscr{L}(\mathscr{A})$ follows from the fact that $\mathscr{L}(\mathscr{A})$ is the orthogonal space to $[\mathscr{A}, \mathscr{A}]$ under $\phi$.

Now let $\mathscr{A}_{c}$ be the complexified algebra of $\mathscr{A}$ and let $\mathscr{A}_{c}=$ $\sum_{i=1}^{n} \oplus \mathscr{A}_{i}$ be the decomposition of $\mathscr{A}_{c}$ into generalized eigenspaces of $\tau$.

For each complex number $\alpha$ let $\mathscr{A}_{\alpha}$ be zero if $\alpha$ is not an eigenvalue and let $\mathscr{A}_{\alpha}$ be the eigenspace corresponding to $\alpha$ if $\alpha$ is an eigenvalue. Each $\mathscr{A}_{\alpha}$ is a left ideal since $\tau$ is a left module homomorphism.

Let $\mathscr{H}=\left(\sum_{\alpha \neq 1, \alpha \neq 0} \mathscr{A}_{\alpha}\right) \cap \mathscr{A}$

$$
\mathscr{C}=\left(\mathscr{A}_{0}+\mathscr{A}_{1}\right) \cap \mathscr{\mathscr { C }} \text {. }
$$

Since 0 is not an eigenvalue of $\tau^{\phi}=I-\tau$ on $\mathscr{H}, \tau^{\phi}$ is invertible on $\mathscr{H}$. Hence $\mathscr{H} \subset \mathscr{L}(\mathscr{A})$. But then

$$
[\mathscr{A}, \mathscr{H}]=\mathscr{A} \mathscr{H} \subset \mathscr{H} \text {. }
$$

Also $[\mathscr{A}, \mathscr{A}] \subset \mathscr{A}_{0}$ which is disjoint from $\mathscr{H}$ so $\mathscr{A} \mathscr{H}=0$ as well. It is also true that $\mathscr{C}$ and $\mathscr{C}$ are orthogonal under $\phi$. In fact, it is easily verified that $\mathscr{A}_{\alpha}$ and $\mathscr{A}_{\beta}$ are orthogonal if $\alpha \neq 1-\beta$. It follows that to split $\mathscr{B}$, it suffices to split $\mathscr{C} \times \mathscr{Z}(\mathscr{B})$ 
and $\mathscr{C} \times \mathscr{Z}(\mathscr{B})$ separalety: But $\mathscr{H} \times \mathscr{Z}(\mathscr{B})$ is a generalized Heisenberg algebra and so is splitable. In $\mathscr{C} \times \mathscr{Z}(\mathscr{B})$, let $\mathscr{S}=\mathscr{A}_{1} \times \mathscr{Z}(\mathscr{B})$ and $\mathscr{C}=\mathscr{A}_{0} \times \mathscr{Z}(\mathscr{B})$. Then $\mathscr{C} \times \mathscr{Z}(\mathscr{B})=$ $\mathscr{S}+\mathscr{M}$ and $\mathscr{S} \cap \mathscr{M}=\mathscr{Z}(\mathscr{B}) \mathscr{C} \supset[\mathscr{A}, \mathscr{A}] \times \mathscr{Z}(\mathscr{B})$ as desired. $\mathscr{S}$ is abelian since $\mathscr{A}_{1}$ is orthogonal to $\mathscr{A}_{1}$ under $\phi$ and $\left[\mathscr{A}_{1}, \mathscr{A}_{1}\right] \subset$ $\mathscr{A}_{1} \cap \mathscr{A}_{0}=\{0\}$.

$\mathscr{A}_{0} \subset \mathscr{L}(\mathscr{A})$ because $\tau^{\phi}$ is invertible on $\mathscr{A}_{0} . \mathscr{K}_{0}$ is also selforthogonal so $\mathscr{C}$ is abelian. To finish our theorem, we must prove that $\mathscr{A}_{0}$ and $\mathscr{A}_{1}$ have the same dimension. However, $\phi$ is nondegenerate on $\mathscr{C} \times \mathscr{C}$ and $\mathscr{A}_{0}$ and $\mathscr{A}_{1}$ are each self-orthogonal. Hence $\mathscr{A}_{1}$ is isomorphic with $\left(\mathscr{A}_{0}\right)^{*}$ under the pairing defined by $\phi$.

REMARK. Although the above theorem proves that for each split nilpotent Lie algebra $\mathscr{N}$, there is an associative algebra $\mathscr{B}$ which gives rise to $\mathscr{N}$, it is not true that this correspondence is one-to-one. In fact, consider the Heisenberg Lie algebra which we take to be $\boldsymbol{R}^{2} \times \boldsymbol{R}$ with the Lie structure:

$$
[(x, s),(y, t)]=\left(0, x_{1} y_{2}-y_{1} x_{2}\right) .
$$

There are two, nonisomorphic left commutative algebra structures on $\boldsymbol{R}^{2} \times \boldsymbol{R}$ which gives rise to this same Lie algebra. They are defined by:

$$
\begin{aligned}
& \text { (i) }(x, s) \cdot(y, t)=\left(0, x_{1} y_{2}\right) \\
& \text { (ii) }(x, s) \cdot(y, t)=\left(0,\left(x_{1} y_{2}-y_{1} x_{2}\right) / 2\right) .
\end{aligned}
$$

They are nonisomorphic since in case (i) the left annihilator is $(0 \times R) \times$ $\boldsymbol{R}$ while in case (ii) the left annihilator is $(0 \times 0) \times \boldsymbol{R}$. For many questions, one might study on the Lie group, the proper selection of the algebra can be important. See, for example, the Fourier transform theorems of [5].

We understand, incidentally, that L. Auslander has also observed these two algebra structures mentioned above.

Theorem I has an interesting consequence which yields information even in the split $H A T$ case. Let $G$ be a locally compact topological group and let $U$ be an irreducible, unitary representation of $G$. Let $K$ be the kernel of $U$. Then $U$ defines an injective representation $\widetilde{U}$ of $G / K$. We shall say that $U$ is square-integrable modulo its kernel if $\widetilde{U}$ has square-integrable matrix elements-i.e., there are vectors $v$ and $w$ such that the function $g \rightarrow(\tilde{V}(g) v, w)$ is nonzero and square-integrable on $G / K$. If $G$ is a Lie group, $G$ is said to be ad-associative if its Lie algebra is ad-associative.

THEOREM III. Let $G$ be an ad-associative, nilpotent Lie group. 
Then every irreducible unitary representation of $G$ is square integrable modulo its kernel.

Proof. Let $\mathscr{L}$ be the Lie algebra of $G$. By Theorem I, $\mathscr{L}$ is $\mathscr{B} / \mathscr{F}$ where $\mathscr{B}$ is a left-commutative algebra and $\mathscr{F}$ is an ideal. By a lifting argument, we may assume that $\mathscr{B}=\mathscr{L}$. Let $U$ be an irreducible, unitary representation of $G$ and let $\lambda \in \mathscr{B}^{*}$ define the Kirillov orbit of $U$ (see [3]). It follows from Moore-Wolf [4] (or, more precisely, from [1]) that $U$ is square-integrable modulo its kernel iff the radical of the form

$$
\mathscr{B}_{\lambda}(x, y)=\lambda([x, y])=\phi(x, y)
$$

is a Lie ideal of $\mathscr{B}$. The form $\mathscr{B}$ is easily seen to be a twine form for $\mathscr{B}$ (perhaps degenerate). The radical $\mathscr{R}_{\lambda}$ of $\mathscr{B}_{\lambda}$ is $\{0\}^{\phi}$ so $\mathscr{R}_{2}$ is infact an algebra ideal from Lemma A.

\section{REFERENCES}

1. J. Brezin, Geometry and the method of Kirillov, in Non-Commutative Harmonic Analysis, Springer \#466, 1975.

2. R. Howe, On a connection between nilpotent groups and oscillatory integrals associated to singularities, Pacific. J. Math., 73 (1977), 329-364.

3. A. Kirillov, Unitary representations of nilpotent Lie groups, Russian Mathematical Surveys, 17 (1962), 53-104.

4. C. C. Moore and J. Wolf, Square integrable representations of nilpotent groups, Trans. Amer. Math. Soc., 185 (1973), 445-462.

5. R. Penney, The Euclidian Fourier transform on nilmanifolds, preprint.

Received January 15, 1980.

Purdue University

West LafayetTe, IN 47907 


\title{
PACIFIC JOURNAL OF MATHEMATICS
}

\section{EDITORS}

\author{
DONALD BABBITT (Managing Editor) \\ University of California \\ Los Angeles, CA 90024 \\ Hugo RossI \\ University of Utah \\ Salt Lake City, UT 84112 \\ C. C. MOORE and Arthur Agus \\ University of California \\ Berkeley, CA 94720
}

\section{J. DugundjI}

Department of Mathematics

University of Southern California

Los Angeles, CA 90007

R. FinN and J. Milgram

Stanford University

Stanford, CA 94305

\section{ASSOCIATE EDITORS}
R. ARENS
E. F. BeCKENBACH
B. H. NeumanN
F. WOLF
K. YoshidA

\section{SUPPORTING INSTITUTIONS}

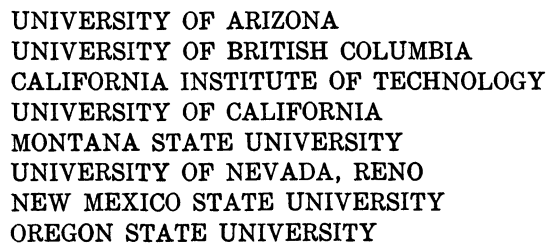

UNIVERSITY OF ARIZONA

UNIVERSITY OF BRITISH COLUMBIA

CALIFORNIA INSTITUTE OF TECHNOLOGY

UNIVERSITY OF CALIFORNIA

MONTANA STATE UNIVERSITY

UNIVERSITY OF NEVADA, RENO

NEW MEXICO STATE UNIVERSITY

OREGON STATE UNIVERSITY

\author{
UNIVERSITY OF OREGON \\ UNIVERSITY OF SOUTHERN CALIFORNIA \\ STANFORD UNIVERSITY \\ UNIVERSITY OF HAWAII \\ UNIVERSITY OF TOKYO \\ UNIVERSITY OF UTAH \\ WASHINGTON STATE UNIVERSITY \\ UNIVERSITY OF WASHINGTON
}

The Supporting Institutions listed above contribute to the cost of publication of this Journal, but they are not owners or publishers and have no responsibility for its content or policies.

Mathematical papers intended for publication in the Pacific Journal of Mathematics should be in typed form or offset-reproduced, (not dittoed), double spaced with large margins. Please do not use built up fractions in the text of the manuscript. However, you may use them in the displayed equations. Underline Greek letters in red, German in green, and script in blue. The first paragraph or two must be capable of being used separately as a synopsis of the entire paper. Please propose a heading for the odd numbered pages of less than 35 characters. Manuscripts, in triplicate, may be sent to any one of the editors. Please classify according to the scheme of Math. Reviews, Index to Vol. 39. Supply name and address of author to whom proofs should be sent. All other communications should be addressed to the managing editor, or Elaine Barth, University of California, Los Angeles, California, 90024.

50 reprints to each author are provided free for each article, only if page charges have been substantially paid. Additional copies may be obtained at cost in multiples of 50 .

The Pacific Journal of Mathematics is issued monthly as of January 1966. Regular subscription rate: $\$ 102.00$ a year (6 Vols., 12 issues). Special rate: $\$ 51.00$ a year to individual members of supporting institutions.

Subscriptions, orders for numbers issued in the last three calendar years, and changes of address shoud be sent to Pacific Journal of Mathematics, P.O. Box 969, Carmel Valley, CA 93924, U.S.A. Old back numbers obtainable from Kraus Periodicals Co., Route 100, Millwood, NY 10546.

\section{PUBLISHED BY PACIFIC JOURNAL OF MATHEMATICS, A NON-PROFIT CORPORATION}

Printed at Kokusai Bunken Insatsusha (International Academic Printing Co., Ltd.). 8-8, 3-chome, Takadanobaba, Shinjuku-ku, Tokyo 160, Japan. 


\section{Pacific Journal of Mathematics}

Vol. 99, No. $2 \quad$ June, 1982

Thomas E. Armstrong and Karel Libor Prikry, On the semimetric on a

Boolean algebra induced by a finitely additive probability measure . . . .249

Walter Russell Bloom, Strict local inclusion results between spaces of

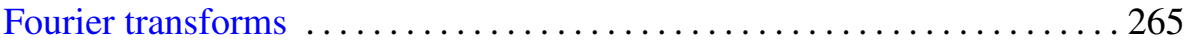

Richard Clark Brown, Notes on generalized boundary value problems in

Banach spaces. II. Infinite-dimensional extension theory ........... 271

Sui Sun Cheng, Isoperimetric eigenvalue problem of even order differential

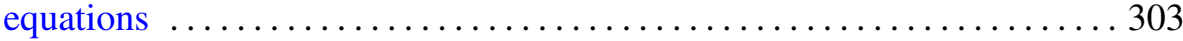

Lung O. Chung and Jiang Luh, Derivations of higher order and

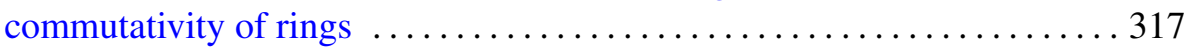

Ali Ahmad Fora, A fixed point theorem for product spaces . . . . . . . . . 327

Barry J. Gardner, Radical classes of regular rings with Artinian primitive

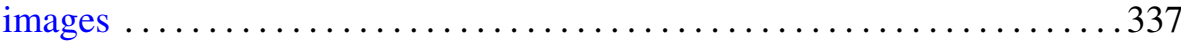

John Brady Garnett and Peter Wilcox Jones, BMO from dyadic BMO . . . 351

Allen E. Hatcher, On the boundary curves of incompressible surfaces . . . . 373

Richard Howard Hudson and Kenneth S. Williams, Resolution of

ambiguities in the evaluation of cubic and quartic Jacobsthal sums .....379

Viktor Losert, Counter-examples to some conjectures about doubly

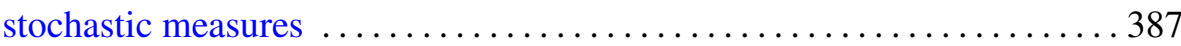

Kenneth Derwood Magill, Jr., P. R. Misra and Udai Bhan Tewari,

Structure spaces for sandwich semigroups

Mark Mandelker, Continuity of monotone functions

Kenneth Guy Miller, An index theorem and hypoellipticity on nilpotent Lie groups ......................................... 419

Evelyn M. Nelson, Homomorphisms of mono-unary algebras . . . . . . . . . 427

Marvin E. Ortel, The support of an extremal dilatation . . .

R. S. Pathak and O. P. Singh, Finite Hankel transforms of distributions . . . 439

Richard Cole Penney, The theory of ad-associative Lie algebras

Linda Ruth Sons, Zero distribution of functions with slow or moderate

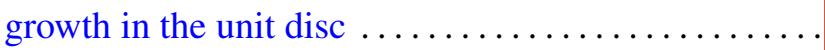

Russell Bruce Walker, Transversals to laminations 